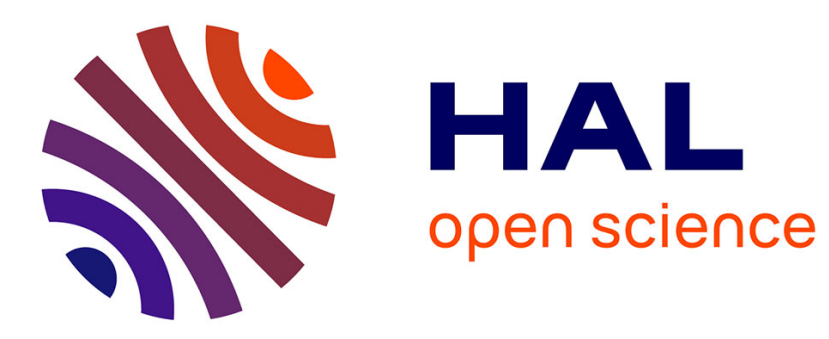

\title{
Flavour production by Saprochaete and Geotrichum yeasts and their close relatives
}

Eric Grondin, Alain Shum Cheong Sing, Steve James, Carmen Nueno-Palop, Jean Marie François, Thomas Petit

\section{- To cite this version:}

Eric Grondin, Alain Shum Cheong Sing, Steve James, Carmen Nueno-Palop, Jean Marie François, et al.. Flavour production by Saprochaete and Geotrichum yeasts and their close relatives. Food Chemistry, 2017, 237, pp.677-684. 10.1016/j.foodchem.2017.06.009 . hal-01606681

\section{HAL Id: hal-01606681 https://hal.science/hal-01606681}

Submitted on 3 Oct 2017

HAL is a multi-disciplinary open access archive for the deposit and dissemination of scientific research documents, whether they are published or not. The documents may come from teaching and research institutions in France or abroad, or from public or private research centers.
L'archive ouverte pluridisciplinaire HAL, est destinée au dépôt et à la diffusion de documents scientifiques de niveau recherche, publiés ou non, émanant des établissements d'enseignement et de recherche français ou étrangers, des laboratoires publics ou privés. 


\section{Flavour production by Saprochaete and Geotrichum yeasts and their close relatives}

Eric Grondin ${ }^{\mathrm{a}, \mathrm{b}}$, Alain Shum Cheong Sing ${ }^{\mathrm{a}}$, Steve James ${ }^{\mathrm{c}}$, Carmen Nueno-Palop ${ }^{\mathrm{c}}$, Jean Marie François ${ }^{\mathrm{d}}$, Thomas Petit ${ }^{\text {a,b,e, } *}$

a Université de La Réunion, Laboratoire de Chimie des Substances Naturelles et des Sciences des Aliments, LCSNSA, EA 2212, 15 Avenue René Cassin, 97490 Sainte Clotilde, La Réunion, France

${ }^{\mathrm{b}}$ Département Génie Biologique, IUT, Université de La Réunion, Saint-Pierre, Ile de la Réunion, France

c National Collection of Yeast Cultures, Institute of Food Research, Norwich Research Park, Colney, Norwich NR4 7UA, UK

d LISBP, Université Fédérale de Toulouse, CNRS, INRA, INSA, 135 Avenue de Rangueil, F-31077 Toulouse, France

e UMR Qualisud - Démarche intégrée pour l'obtention d'aliments de qualité, Université de La Réunion, IUT -

Saint-Pierre, La Réunion, France

\section{A B S T R A C T}

In this study, a total of 30 yeast strains belonging to the genera Dipodascus, Galactomyces, Geotrichum, Magnusiomyces and Saprochaete were investigated for volatile organic compound production using HSSPME-GC/MS analysis. The resulting flavour profiles, including 36 esters and 6 alcohols compounds, were statistically evaluated by cluster and PCA analysis. Two main groups of strains were extracted from this analysis, namely a group with a low ability to produce flavour and a group producing mainly alcohols. Two other minor groups of strains including Saprochaete suaveolens, Geotrichum marinum and Saprochaete gigas were diverging significantly from the main groups precisely because they showed a good ability to produce a large diversity of esters. In particular, we found that the Saprochaete genus (and their closed relatives) was characterized by a high production of unsaturated esters arising from par-tial catabolism of branched chain amino-acids. These esters were produced by eight phylogenetically related strains of Saprochaete genus.

\section{Introduction}

Geotrichum and Saprochaete yeasts are filamentous yeast like fungi which belong to the Ascomycota division, Saccharomycetes class and Saccharomycetales order (De Hoog \& Smith, 2011a, 2011b). Saprochaete and Geotrichum species are very closely related. This is best illustrated by the fact that some species like Saprochaete suaveolens and Saprochaete clavata have a separate Geotrichum name (synonym) (De Hoog \& Smith, 2004). These microbial eukaryotes are cosmopolitan and widespread and are often found in soil, manure, fruits, dairy products, human skin and digestive tract, insects as well as in other environments such as decaying plants and industrial effluents (Damasceno, Cereda, Pastore, \& Oliveira, 2003; Suh \& Blackwell, 2006). De Hoog and Smith (2011a) listed 11 species of Geotrichum of which one has a

\footnotetext{
* Corresponding author at: UMR Qualisud, Université de La Réunion, 40 Avenue de Soweto, 97410 Saint Pierre, La Réunion, France.

E-mail address: thomas, petit@univ-reunion,fr (T. Petit).
}

teleomorphic state in the genus Dipodascus de Lagerh, three in the genus Galactomyces Redhead and Malloch and seven for which a sexual state has not been found. G. ghanense (Nielsen, Jakobsen, \& Jespersen, 2010) was the most recent Geotrichum species found in nature. In the Saprochaete genus, 13 species were described by De Hoog and Smith (2011b) of which, only three have a teleomorphic state in the genus Magnusiomyces. Within this genus, some species like $S$. suaveolens, S. clavata, S. gigas and $S$. ingens have their syn onym in the Geotrichum genus (G. fragrans, G. clavatum, G. gigas and $G$. ingens, respectively). With respect to safety, strains such as Saprochaete capitata, S. clavata or G. candidum are described as human pathogens (Camus et al., 2014; Garcia Ruiz et al., 2013).

Geotrichum candidum is one of the most studied species among this group of yeasts. It is well known in food industry for cheese ripening (Marcellino, Beuvier, Grappin, Guéguen, \& Benson, 2001) and enzyme production (Ayed, Assas, Sayadi, \& Hamdi, 2005; Brabcova et al., 2013; Hang \& Woodams, 1990). Geotrichum can didum, as well as S. suaveolens, G. klebahnii, Gal. geotrichum, Gal. reessii, D. aggregatus, D. albidus D. armillariae, M. capitatus and $M$. 
magnusii are also well known for flavour production (Bonnarme et al., 2001; Buzzini, Martini, Cappelli, Pagnoni, \& Davoli, 2003; Damasceno et al., 2003; De Oliveira et al., 2013; Farbood, Morris, \& Seitz, 1987; Fischer, Senser, \& Grosch, 1983; Jollivet, Chataud, Vayssier, Bensoussan, \& Belin, 1994; Neto, Pastore, \& Macedo, 2004; Shimizu, Kataoka, Kizaki, \& Yasohara, 2004; Sinha, 2007; $\mathrm{Wu}, \mathrm{Xu}$, \& Chen, 2012).

Flavours are secondary metabolites produced by living cells through some specific pathways such as Ehrlich (Hazelwood, Daran, van Maris, Pronk, \& Dickinson, 2008), $\beta$ oxidation (Maggio Hall \& Keller, 2004) and glycolytic pathways (Liu, Holland, \& Crow, 2004; Ugliano \& Henschke, 2009). Among yeasts, Saccharomyces cerevisiae is probably the best know species for its applications in the field of food flavouring (Saerens, Delvaux, Verstrepen, \& Thevelein, 2010). Other non conventional yeasts, like Saprochaete suaveolens, have also been described as excellent producers of fla vours including unsaturated esters such as ethyl tiglate (ethyl $(E)$ 2 methylbut 2 enoate), an interesting top note flavour character ized by a strong fruity odor (Grondin, Shum Cheong Sing, Caro, de Billerbeck, et al., 2015; Grondin, Shum Cheong Sing, Caro, Raherimandimby, et al., 2015). Numerous publications deal with the production of flavours from both an academic and applied per spective, but comparatively few of them uses flavours as a tool to discriminate between species and/or strains. Chemotaxonomy, according to the definition given by Frisvad, Andersen, and Thrane (2008) using flavours as a taxonomic tool was first reported for the filamentous fungi Penicillium (Larsen \& Frisvad, 1995).

The purpose of this study was to determine the flavour produc tion profiles of the Saprochaete, Geotrichum and closely related yeasts. Experimental measurement of flavour production profiles using HS SPME CG/SM and processing of the data using descriptive statistic methods were performed to characterise the volatile organic compounds (VOCs) of these yeasts. Multivariate statistical methods were applied to find out whether the flavours production by these yeast species and their genomic classification using the ribosomal internal transcribed spacer (ITS) sequences were correlated.

\section{Materials and methods}

\subsection{Yeast strains}

The list of strains used in this study is presented in Table 1. Most of them were purchased from CBS (Utrecht, The Netherlands) and from BCCM (Brussels, Belgium) strain collections. The Saprochaete suaveolens strain (GEC 0) used in this study was isolated from Pitaya fruits collected in Reunion Island as described elsewhere (Grondin, Shum Cheong Sing, Caro, de Billerbeck, et al., 2015; Grondin, Shum Cheong Sing, Caro, Raherimandimby, et al., 2015). The rDNA D1/D2 sequence of the isolated strain showed a difference of one nucleotide with the G. fragrans reference strain CBS 152.25.

\subsection{Culture media}

Cells were stored in a rich medium contening glycerol in cryo genic vials at $80^{\circ} \mathrm{C}$ and refreshed on autoclaved YEPD agar slants containing $20 \mathrm{~g} / \mathrm{L}$ of glucose (Sigma), $20 \mathrm{~g} / \mathrm{L}$ of peptone (Becton, Dickinson and Co.), $10 \mathrm{~g} / \mathrm{L}$ of yeast extract (Biokar Diagnostics) and $15 \mathrm{~g} / \mathrm{L}$ of agar (Merck). Cells were refreshed at $28^{\circ} \mathrm{C}$ for $48 \mathrm{~h}$ prior to their utilization.

\subsection{VOCS analysis}

Isolation and characterization of volatile metabolites was per formed using solid phase micro extraction (SPME), followed by
Table 1

Strains of Saprochaete, Geotrichum and teleomorphs used in this study.

\begin{tabular}{|c|c|c|c|}
\hline $\begin{array}{l}\text { Strain } \\
\text { number }\end{array}$ & Species & $\begin{array}{l}\text { Collection } \\
\text { number }\end{array}$ & $\begin{array}{l}\text { GenBank accession } \\
\text { number of ITS } \\
\text { sequences }\end{array}$ \\
\hline S1 & Dipodascus albidus & CBS 766.85 & AY788342 \\
\hline S2 & Dipodascus armilliare & CBS 834.71 & AY788350.1 \\
\hline S3 & Galactomyces candidus & CBS 11176 & KJ579946 \\
\hline S4 & $\begin{array}{l}\text { Galactomyces citri- } \\
\text { aurantii }\end{array}$ & CBS 176.89 & AY788296.1 \\
\hline S5 & Galactomyces geotrichum & CBS 774.71 & JN974293.1 \\
\hline S6 & $\begin{array}{l}\text { Galactomyces } \\
\text { pseudocandidus }\end{array}$ & CBS 10073 & JN974292.1 \\
\hline S7 & Geotrichum candidum & CBS 615.84 & $\mathrm{KJ} 608128^{\mathrm{a}}$ \\
\hline S8 & Geotrichum carabidarum & CBS 9891 & DQ143888.1 \\
\hline S9 & $\begin{array}{l}\text { Geotrichum } \\
\text { cucujoidarum }\end{array}$ & CBS 9893 & DQ143890 \\
\hline S10 & Geotrichum eriense & CBS 694.83 & This study \\
\hline S11 & Geotrichum europaeum & CBS 866.68 & AY788351 \\
\hline S12 & Geotrichum fermentans & CBS 625.85 & AY788319 \\
\hline S13 & Geotrichum ghanense & CBS 11010 & This study \\
\hline S14 & Geotrichum histeridarum & CBS 9892 & DQ143889 \\
\hline S15 & Geotrichum klebahnii & CBS 179.30 & AY788298 \\
\hline S16 & Geotrichum marinum & $\begin{array}{l}\text { MUCL } \\
42958\end{array}$ & This study \\
\hline S17 & Geotrichum phurueaensis & CBS 11418 & HE663403 \\
\hline S18 & Geotrichum restrictum & $\begin{array}{l}\text { CBS } \\
111234\end{array}$ & EF126738.1 \\
\hline S19 & Magnusiomyces ingens & $\begin{array}{l}\text { CBS } \\
101346\end{array}$ & This study \\
\hline S20 & Magnusiomyces magnusii & CBS 151.30 & AY788290.1 \\
\hline S21 & Magnusiomyces ovetensis & CBS 192.55 & AY788303.1 \\
\hline S22 & Saprochaete chiloensis & CBS 8187 & AY788349 \\
\hline S23 & Saprochaete fungicola & CBS 625.85 & AY788333.1 \\
\hline S24 & Saprochaete gigas & CBS 126.76 & AY838940.1 \\
\hline S25 & Saprochaete ingens & CBS 524.90 & AY788326.1 \\
\hline S26 & Saprochaete japonica & $\begin{array}{l}\text { CBS } \\
100158\end{array}$ & AY788287.1 \\
\hline S27 & Saprochaete psychrophila & CBS 765.85 & AY788341.1 \\
\hline S28 & Saprochaete quercus & CBS 752.85 & This study \\
\hline S29 & $\begin{array}{l}\text { Saprochaete } \\
\text { saccharophila }\end{array}$ & CBS 412.95 & AY788316.1 \\
\hline S30 & Saprochaete suaveolens & GEC0 & This study \\
\hline
\end{tabular}

a Sequence of another strains of the same species.

gas chromatography mass spectrometry (GC MS) analysis. The VOCS extraction and the chromatographic conditions were described in our previous studies (Grondin, Shum Cheong Sing, Caro, de Billerbeck, et al., 2015; Grondin, Shum Cheong Sing, Caro, Raherimandimby, et al., 2015).

\subsection{Phylogenetic analysis}

The ribosomal internal transcribed spacer (ITS) sequences were retrieved from GenBank (NCBI database) under the accession num bers indicated in Table 1 . Where sequences were not available in the database, analysis was performed according to James et al. (2014). Yeast cells were breaked using microwaves (Panasonic, $800 \mathrm{~W}$ ) for $30 \mathrm{~s}$ in $50 \mu \mathrm{L}$ of water to obtain cells extracts. The ITS region was amplified by PCR directly from whole yeast cell extracts, amplified using primers ITS5 and ITS4 and sequenced using these primers as well as internal primers ITS2 and ITS3. The amplified DNA was checked by $1 \%$ agarose gel electrophoresis, purified and concentrated using QIAquick PCR purification spin columns (Qiagen) and sequenced using a Life Technologies 3730XL sequencer at the Genome Analysis Centre (TGAC), Nor wich, UK. Pairwise alignments and phylogenetic analysis were con ducted using Geneious 7.1 software created by Biomatters. A phylogenetic neighbour joining tree was then generated using the distance Tamura Nei model. Confidence values for branch nodes were estimated from bootstrap analyses of 1000 replicates 
Table 2

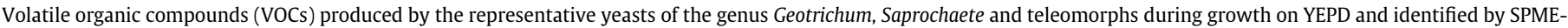
GC/MS.

\begin{tabular}{|c|c|c|c|}
\hline Volatils compounds & $\begin{array}{l}\text { RRI } \\
\text { EXP }^{\mathrm{a}}\end{array}$ & $\begin{array}{l}\text { RRI } \\
\mathrm{TH}^{\mathrm{b}}\end{array}$ & Yeast producing strains \\
\hline \multicolumn{4}{|l|}{ Alcohol } \\
\hline 2-Methylpropan-1-ol (isobutanol) & 637 & 628 & G. pseudocandidus; G. europaeum; S. fungicola \\
\hline Butan-1-ol & 661 & 662 & G. candidum; G. carabidarum; G. restrictum; M. magnusii; S. suaveolens \\
\hline 2-Methylbutanol (active amyl alcohol) & 770 & 739 & $\begin{array}{l}\text { G. pseudocandidus; G. candidum; G. cucujoidarum; G. europaeum; G. histeridarum; G. marinum; G. restrictum; } \\
\text { M. magnusii; S. fungicola; S. quercus; S. suaveolens }\end{array}$ \\
\hline 3-Methylbutanol (isoamyl alcohol) & 731 & 734 & $\begin{array}{l}\text { D. armilliare; G. candidus; G. citri-aurantii; G. geotrichum; G. pseudocandidus; G. candidum; G. cucujoidarum; G. } \\
\text { europaeum; G. fermentans; G. histeridarum; G. marinum; G. phurueaensis; G. restrictum; M. magnusii; S. } \\
\text { fungicola; S. gigas; S. japonica; S. quercus; S. suaveolens }\end{array}$ \\
\hline 2-Phenylethanol & 1105 & 1114 & $\begin{array}{l}\text { G. candidus; G. citri-aurantii; G. pseudocandidus; G. candidum; G. eriense; G. europaeum; G. fermentans; G. } \\
\text { ghanense; G. histeridarum; G. marinum; G. phurueaensis; M. magnusii; M. ovetensis; S. fungicola; S. gigas; S. } \\
\text { japonica; S. quercus; S. suaveolens }\end{array}$ \\
\hline 2-Ethylhexanol & 1021 & 1030 & G. histeridarum; M. magnusii; S. fungicola; S. quercus \\
\hline \multicolumn{4}{|r|}{ 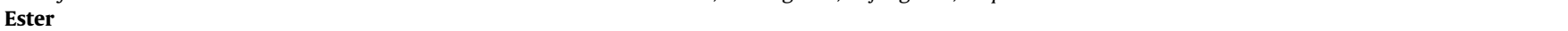 } \\
\hline Methyl 2-methylbutanoate & 774 & & G. marinum \\
\hline $\begin{array}{l}\text { Methyl 3-methylbutanoate (methyl } \\
\text { isovalerate) }\end{array}$ & 774 & & G. pseudocandidus \\
\hline Ethyl ethanoate (ethyl acetate) & 620 & 612 & G. carabidarum; G. europaeum; G. marinum; G. restrictum; S. quercus; S. suaveolens \\
\hline Ethyl propanoate & 709 & 709 & G. marinum; S. gigas; S. suaveolens \\
\hline $\begin{array}{l}\text { Ethyl 2-methylpropanoate (ethyl } \\
\text { isobutyrate) }\end{array}$ & 755 & 756 & G. pseudocandidus; G. europaeum; G. histeridarum; G. marinum; S. gigas; S. suaveolens \\
\hline Ethyl butanoate & 797 & 800 & G. pseudocandidus; G. candidum; G. histeridarum; G. marinum; G. restrictum; S. suaveolens \\
\hline Ethyl but-2-enoate (ethyl crotonate) & 841 & 833 & G. marinum; S. suaveolens \\
\hline Ethyl 2-methylbutanoate & 847 & 846 & G. pseudocandidus; G. candidum; G. europaeum; G. histeridarum; G. marinum; S. suaveolens \\
\hline $\begin{array}{l}\text { Ethyl (E)-2-methylbut-2-enoate (ethyl } \\
\text { tiglate) }\end{array}$ & 935 & 936 & G. geotrichum; G. pseudocandidus; G. europaeum; G. marinum; M. magnusii; S. gigas; S. quercus; S. suaveolens \\
\hline $\begin{array}{l}\text { Ethyl 3-methylbutanoate (ethyl } \\
\text { isovalerate) }\end{array}$ & 850 & 849 & $\begin{array}{l}\text { G. geotrichum; G. pseudocandidus; G. candidum; G. europaeum; G. fermentans; G. marinum; S. gigas; S. } \\
\text { suaveolens }\end{array}$ \\
\hline $\begin{array}{l}\text { Ethyl (E)-3-methylbut-2-enoate (ethyl 3- } \\
\text { methylcrotonate) }\end{array}$ & 919 & 920 & G. marinum; S. gigas; S. suaveolens \\
\hline Ethyl pentanoate (ethyl valerate) & 895 & & G. marinum \\
\hline Ethyl hexanoate (ethyl caproate) & 991 & & G. marinum \\
\hline Ethyl hex-2-enoate & 1037 & & G. marinum \\
\hline Ethyl octanoate (ethyl caprylate) & 1186 & & G. carabidarum; G. restrictum; S. quercus \\
\hline $\begin{array}{l}\text { 2-Methylpropyl ethanoate (isobutyl } \\
\text { acetate) }\end{array}$ & 771 & 753 & S. suaveolens \\
\hline $\begin{array}{l}\text { 2-Methylpropyl 2-methylpropanoate } \\
\text { (isobutyl isobutyrate) }\end{array}$ & 909 & 910 & G. marinum; S. gigas; S. suaveolens \\
\hline $\begin{array}{l}\text { 2-Methylpropyl butanoate (isobutyl } \\
\text { butyrate) }\end{array}$ & 951 & 950 & G. marinum; S. suaveolens \\
\hline $\begin{array}{l}\text { 2-Methylpropyl 2-methylbutanoate } \\
\text { (isobutyl 2-methylbutanoate) }\end{array}$ & 995 & 998 & G. marinum; S. gigas; S. suaveolens \\
\hline $\begin{array}{l}\text { 2-Methylpropyl (E)-2-methylbut-2- } \\
\text { enoate (isobutyl tiglate) }\end{array}$ & 1084 & 1086 & G. europaeum; G. marinum; S. gigas; S. suaveolens \\
\hline $\begin{array}{l}\text { 2-Methylpropyl 3-methylbutanoate } \\
\text { (isobutyl isovalerate) }\end{array}$ & 997 & 1002 & G. pseudocandidus; G. marinum; S. gigas; S. suaveolens \\
\hline Butyl 2-methylbutanoate & 1035 & & S. suaveolens \\
\hline $\begin{array}{l}\text { Butyl (E)-2-methylbut-2-enoate (butyl } \\
\quad \text { tiglate) }\end{array}$ & 1125 & 1128 & S. suaveolens \\
\hline $\begin{array}{l}\text { Butyl 3-methylbutanoate (butyl } \\
\text { isovalerate) }\end{array}$ & 1039 & 1040 & S. gigas; S. suaveolens \\
\hline 2-Methylbutyl ethanoate & 903 & 874 & S. suaveolens \\
\hline 2-Methylbutyl 2-methylpropanoate & 1009 & & G. marinum; S. suaveolens \\
\hline 2-Methylbutyl butanoate & 1052 & 1052 & G. marinum; S. suaveolens \\
\hline $\begin{array}{l}\text { 3-Methylbutyl ethanoate (isoamyl } \\
\text { acetate) }\end{array}$ & 873 & 871 & G. candidum; G. histeridarum; G. marinum; S. suaveolens \\
\hline $\begin{array}{l}\text { 3-Methylbutyl propanoate (isoamyl } \\
\text { propanoate) }\end{array}$ & 964 & 964 & G. marinum; S. suaveolens \\
\hline $\begin{array}{l}\text { 3-Methylbutyl butanoate (isoamyl } \\
\text { butanoate) }\end{array}$ & 1049 & 1050 & G. marinum; S. suaveolens \\
\hline $\begin{array}{l}\text { 3-Methylbutyl 2-methylbutanoate } \\
\text { (isoamyl 2-methylbutanoate) }\end{array}$ & 1091 & & G. marinum; S. suaveolens \\
\hline $\begin{array}{l}\text { 3-Methylbutyl }(E)-2 \text {-methylbut-2-enoate } \\
\text { (isoamyl tiglate) }\end{array}$ & 1185 & 1253 & G. marinum; S. suaveolens \\
\hline $\begin{array}{l}\text { 3-Methylbutyl 3-methylbutanoate } \\
\text { (isoamyl isovalerate) }\end{array}$ & 1096 & 1101 & G. citri-aurantii; G. fermentans; G. marinum; S. gigas; S. suaveolens \\
\hline Pentyl propanoate (amyl propanoate) & 964 & 964 & S. gigas \\
\hline $\begin{array}{l}\text { Pentyl 3-methylbutanoate (amyl } \\
\text { isovalerate) }\end{array}$ & 1098 & 1103 & G. marinum; S. gigas; S. suaveolens \\
\hline Octyl ethanoate & 1199 & & G. carabidarum; G. restrictum \\
\hline
\end{tabular}

a Relative Retention index on non-polar column determined experimentally.

b Relative Retention index on non-polar column (http://webbook.nist.gov/chemistry/). 
Table 3

Classification of the VOCs produced by the strains belonging to the genus Geotrichum, Saprochaete and teleomorphs.

\begin{tabular}{|c|c|c|c|c|c|c|c|c|c|c|c|c|c|c|}
\hline \multirow[t]{2}{*}{ Name of species } & \multicolumn{3}{|c|}{$\begin{array}{l}\text { Concentration of VOCs per } \\
\text { chemical class }(\mu \mathrm{g} / \mathrm{L})\end{array}$} & \multicolumn{3}{|c|}{$\begin{array}{l}\text { Number of VOCs per } \\
\text { chemical class }\end{array}$} & \multicolumn{8}{|c|}{ Number of VOCs per type of hypothetical pathway } \\
\hline & {$[\mathrm{Al}]$} & [Es] & [Ctotal] & Al & Es & Total & GP & MP & PP & $\mathrm{BP}$ & Pep & B1P & $\mathrm{B} 2 \mathrm{P}$ & EP \\
\hline Dipodascus albidus & nd & nd & nd & 0 & 0 & 0 & 0 & 0 & 0 & 0 & 0 & 0 & 0 & 0 \\
\hline Dipodascus armilliare & 5 & nd & 5 & 1 & 0 & 0 & 0 & 0 & 0 & 0 & 0 & 0 & 0 & 1 \\
\hline Galactomyces candidus & 85 & $\mathrm{Nd}$ & 85 & 2 & 0 & 2 & 0 & 0 & 0 & 0 & 0 & 0 & 0 & 2 \\
\hline Galactomyces citri-aurantii & 48 & 7 & 55 & 2 & 1 & 3 & 0 & 0 & 0 & 0 & 0 & 0 & 0 & 4 \\
\hline Galactomyces geotrichum & 9 & 39 & 49 & 1 & 2 & 3 & 2 & 0 & 0 & 0 & 0 & 0 & 1 & 3 \\
\hline Galactomyces pseudocandidus & 305 & 37 & 342 & 4 & 7 & 11 & 5 & 1 & 0 & 1 & 0 & 0 & 1 & 11 \\
\hline Geotrichum candidum & 437 & 8 & 445 & 4 & 4 & 8 & 4 & 0 & 0 & 2 & 0 & 0 & 0 & 6 \\
\hline Geotrichum carabidarum & 46 & 121 & 167 & 1 & 3 & 4 & 3 & 0 & 0 & 1 & 0 & 1 & 0 & 0 \\
\hline Geotrichum cucujoidarum & 75 & nd & 75 & 2 & 0 & 2 & 0 & 0 & 0 & 0 & 0 & 0 & 0 & 2 \\
\hline Geotrichum eriense & 11 & nd & 11 & 1 & 0 & 1 & 0 & 0 & 0 & 0 & 0 & 0 & 0 & 1 \\
\hline Geotrichum europaeum & 1164 & 165 & 1329 & 4 & 6 & 10 & 5 & 0 & 0 & 0 & 0 & 0 & 2 & 10 \\
\hline Geotrichum fermentans & 82 & 11 & 93 & 2 & 2 & 4 & 1 & 0 & 0 & 0 & 0 & 0 & 0 & 5 \\
\hline Geotrichum ghanense & 14 & nd & 14 & 1 & 0 & 0 & 0 & 0 & 0 & 0 & 0 & 0 & 0 & 1 \\
\hline Geotrichum histeridarum & 247 & 30 & 277 & 4 & 4 & 8 & 4 & 0 & 0 & 1 & 0 & 1 & 0 & 6 \\
\hline Geotrichum Klebahnii & nd & nd & nd & 0 & 0 & 0 & 0 & 0 & 0 & 0 & 0 & 0 & 0 & 0 \\
\hline Geotrichum marinum & 375 & 3159 & 3534 & 3 & 27 & 30 & 13 & 1 & 2 & 5 & 1 & 2 & 5 & 29 \\
\hline Geotrichum phurueaensis & 109 & $\mathrm{Nd}$ & 109 & 2 & 0 & 2 & 0 & 0 & 0 & 0 & 0 & 0 & 0 & 2 \\
\hline Geotrichum restrictum & 244 & 169 & 414 & 3 & 4 & 7 & 4 & 0 & 0 & 2 & 0 & 2 & 0 & 2 \\
\hline Magnusiomyces ingens & nd & nd & nd & 0 & 0 & 0 & 0 & 0 & 0 & 0 & 0 & 0 & 0 & 0 \\
\hline Magnusiomyces magnusii & 151 & 1 & 152 & 5 & 1 & 6 & 1 & 0 & 0 & 1 & 0 & 1 & 1 & 4 \\
\hline Magnusiomyces ovetensis & 13 & nd & 13 & 1 & 0 & 0 & 0 & 0 & 0 & 0 & 0 & 0 & 0 & 1 \\
\hline Saprochaete chiloensis & nd & nd & nd & 0 & 0 & 0 & 0 & 0 & 0 & 0 & 0 & 0 & 0 & 0 \\
\hline Saprochaete fungicola & 238 & nd & 238 & 5 & 0 & 5 & 0 & 0 & 0 & 0 & 0 & 1 & 0 & 4 \\
\hline Saprochaete gigas & 48 & 1753 & 1801 & 2 & 13 & 15 & 5 & 0 & 1 & 1 & 1 & 0 & 3 & 19 \\
\hline Saprochaete ingens & nd & nd & nd & 0 & 0 & 0 & 0 & 0 & 0 & 0 & 0 & 0 & 0 & 0 \\
\hline Saprochaete japonica & 47 & nd & 47 & 2 & 0 & 2 & 0 & 0 & 0 & 0 & 0 & 0 & 0 & 2 \\
\hline Saprochaete psychrophila & nd & nd & nd & 0 & 0 & 0 & 0 & 0 & 0 & 0 & 0 & 0 & 0 & 0 \\
\hline Saprochaete quercus & 79 & 24 & 103 & 4 & 3 & 7 & 4 & 0 & 0 & 0 & 0 & 2 & 1 & 4 \\
\hline Saprochaete saccharophila & nd & nd & nd & 0 & 0 & 0 & 0 & 0 & 0 & 0 & 0 & 0 & 0 & 0 \\
\hline Saprochaete suaveolens & 268 & 3464 & 3732 & 4 & 28 & 32 & 12 & 0 & 2 & 9 & 1 & 0 & 5 & 33 \\
\hline
\end{tabular}

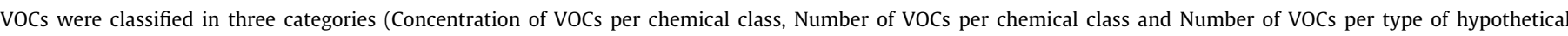

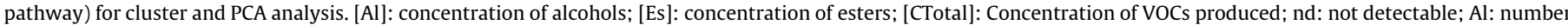

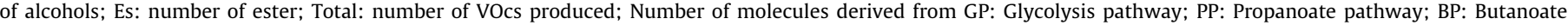
pathway; Pep: Pentanoate pathway; B1P: $\beta$-oxidation pathway; B2P: unsaturated compounds from $\beta$-oxidation pathway; EP: Ehrlich pathway.

(Cardinali et al., 2012; Urubschurov, Janczyk, Souffrant, Freyer, \& Zeyner, 2011).

\subsection{Statistical analysis}

Principal Component Analysis and cluster analysis (Ward's method) were performed on yeast flavours using XLSTAT (Addin soft) (Grondin, Shum Cheong Sing, Caro, de Billerbeck, et al., 2015; Grondin, Shum Cheong Sing, Caro, Raherimandimby, et al., 2015).

\section{Results and discussion}

\subsection{Yeast strain selection}

Yeasts were selected in accordance to De Hoog and Smith (2011a, 2011b) who described all currently accepted yeast species in these genera. Saprochaete clavata, which is categorized as a Class 2 microorganism, was arbitrarily withdrawn because of associated safety problems. VOCs analysis was extended to some strains of Geotrichum, which are not described by De Hoog and Smith (2011a, 2011b) namely G. marinum, G. phuruaensis and G. ghanense. Given that anamorphic and teleomorphic species are genetically very close (Liu et al., 2001), we also selected some yeasts which have an anamorphic state in the genera Geotrichum and Saprochaete, such as Galactomyces citri aurantii (Geotrichum citri aurantii), Gal. pseudocandidus (Geotrichum pseudocandidum), Gal. candidus (Geotrichum candidum), Magnusiomyces magnusii (Saprochaete ludwigii), M. capitatus (Saprochaete capitata), M. ovetensis (Saprochaete sericea) and Dipodascus armillariae (Geotri chum decipiens).

\subsection{VOCS production by different yeast species}

Qualitative analysis of VOCs were carried out using HS SPME GC/MS on 30 representative strains belonging to the anamorphic genera Saprochaete ( 9 species), Geotrichum (12 species) and related teleomorphic genera Magnusiomyces (3 species), Dipodascus (2 spe cies) and Galactomyces ( 4 species) after $24 \mathrm{~h}$ growth on YEPD (Table 2). A total of 42 different compounds were identified and classified into alcohols and esters ( 6 and 36 molecules, respec tively). Due to their aromatic properties, esters are valuable mole cules and are of relevance to industry. These molecules usually impart a characteristic fruity note to fermented beverages such as beer and wine (Schrader, 2007).

Among VOCs, 3 methylbutanol, 2 phenylethanol and 2 methylbutanol, which arise from the degradation of leucine, phenylalanine and isoleucine respectively by the Ehrlich pathway (Hazelwood et al., 2008), were the most frequently encountered compounds and were produced by 19, 18 and 11 different strains, respectively. In contrast, some VOCs such as methyl 2 methylbutanoate and butyl 2 methylbut 2 enoate were produced by only 1 yeast strain (G. marinum and S. suaveolens, respectively). Strains S. suaveolens, G. marinum, S. gigas and G. europaeum showed the best ability to produce qualitatively (more than 10 different compounds) and quantitatively (relative concentration higher than $1000 \mu \mathrm{g} / \mathrm{L}$ ) flavour compounds (Table 3). Strain Gal. pseudocandidus was also found to produce a large number of VOCs ( 11 compounds) but quantitatively, the overall production of VOCs was significantly 


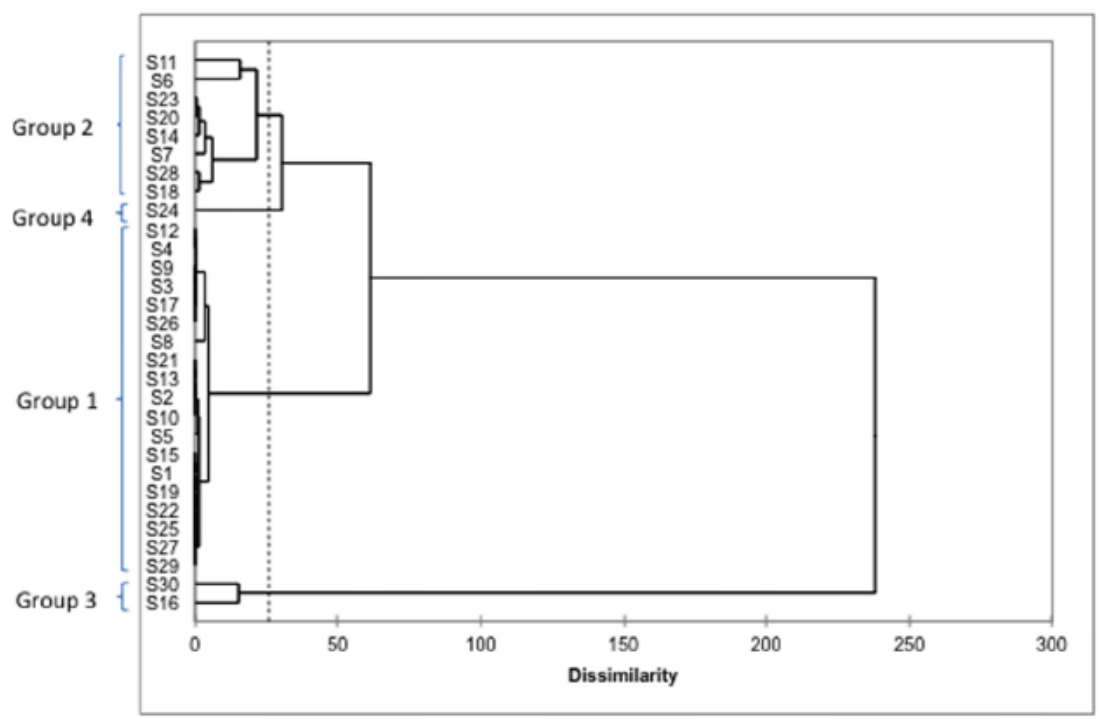

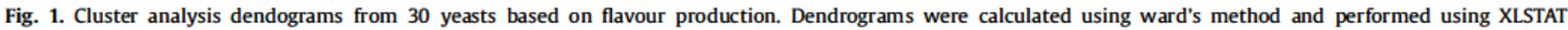
(Addinsoft). Based on entropy, automatic truncation (dotted line) allowed identifying four consistent groups of yeasts among the strains $\mathrm{S} 1$ to $\mathrm{S} 30$ (Table 1 ).

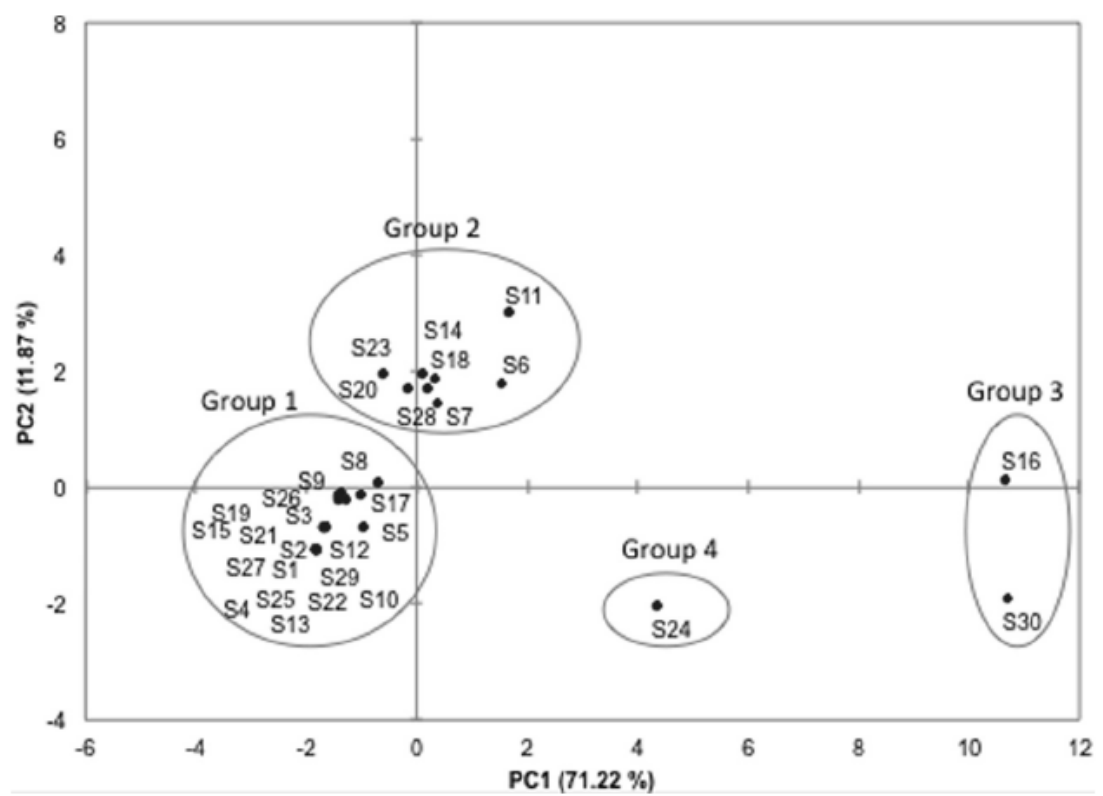

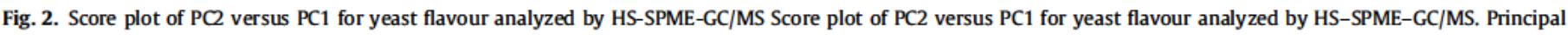

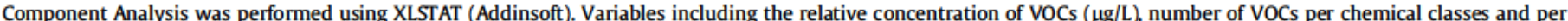

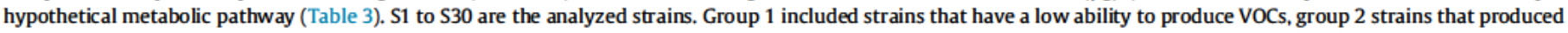
mainly alcohol and Group 3 and 4 strains that produced mainly esters.

lower than the above strains (less than $400 \mu \mathrm{g} / \mathrm{L}$ ). Comparatively, our results were in good agreement with other published data. For instance, production of 2 methylbutanol, 3 methylbutanol, 2 phenylethanol, ethyl acetate, ethyl propanoate, 3 methylbutyl ethanoate, ethyl 2 methylbut 2 enoate and 2 methylbutyl ethano ate were also detected in G. candidum, S. suaveolens, G. klebahnii, Gal. geotrichum and M. magnusii (Buzzini et al., 2003; Damasceno et al., 2003; Farbood et al., 1987; Fischer et al., 1983; Jollivet et al., 1994; Sinha, 2007; Wu et al., 2012). However, some results differed from other published data. Buzzini et al. (2003) reported a production of 3 methylbutanol and pentan 1 ol for G. klebanhii (S13) during growth in shaked flasks containing $10 \mathrm{~g} / \mathrm{L}$ yeast extract, $10 \mathrm{~g} / \mathrm{L}\left(\mathrm{NH}_{4}\right)_{2} \mathrm{HPO}_{4}$ and $20 \mathrm{~g} / \mathrm{L}$ glucose $(\mathrm{pH} 5.0)$ at $25^{\circ} \mathrm{C}$ for $72 \mathrm{~h}$. In our study (YEPD standard agar medium), no VOCs could be detected for this strain. This apparent contradiction might either come from differences in the design of the experiment (e.g., liquid vs. solid media, presence of phosphate buffer, ...) or from the genetic background of the strains. It should be noted however that one of the five G. klebanhii strains isolated by Buzzini et al. (2003) was also unable to produce VOCs. Such a dif ference between strains from the same species was previously reported, for example by Berger, Khan, Molimard, Martin, and Spinnler (1999) who observed differences in the production of sul phur compounds by ten isolates of the same species, G. candidum.

As previously shown by Grondin, Shum Cheong Sing, Caro, de Billerbeck, et al. (2015), Grondin, Shum Cheong Sing, Caro, Raherimandimby, et al. (2015), Saprochaete suaveolens produced several unsaturated ester compounds such as ethyl but 2 enoate, 


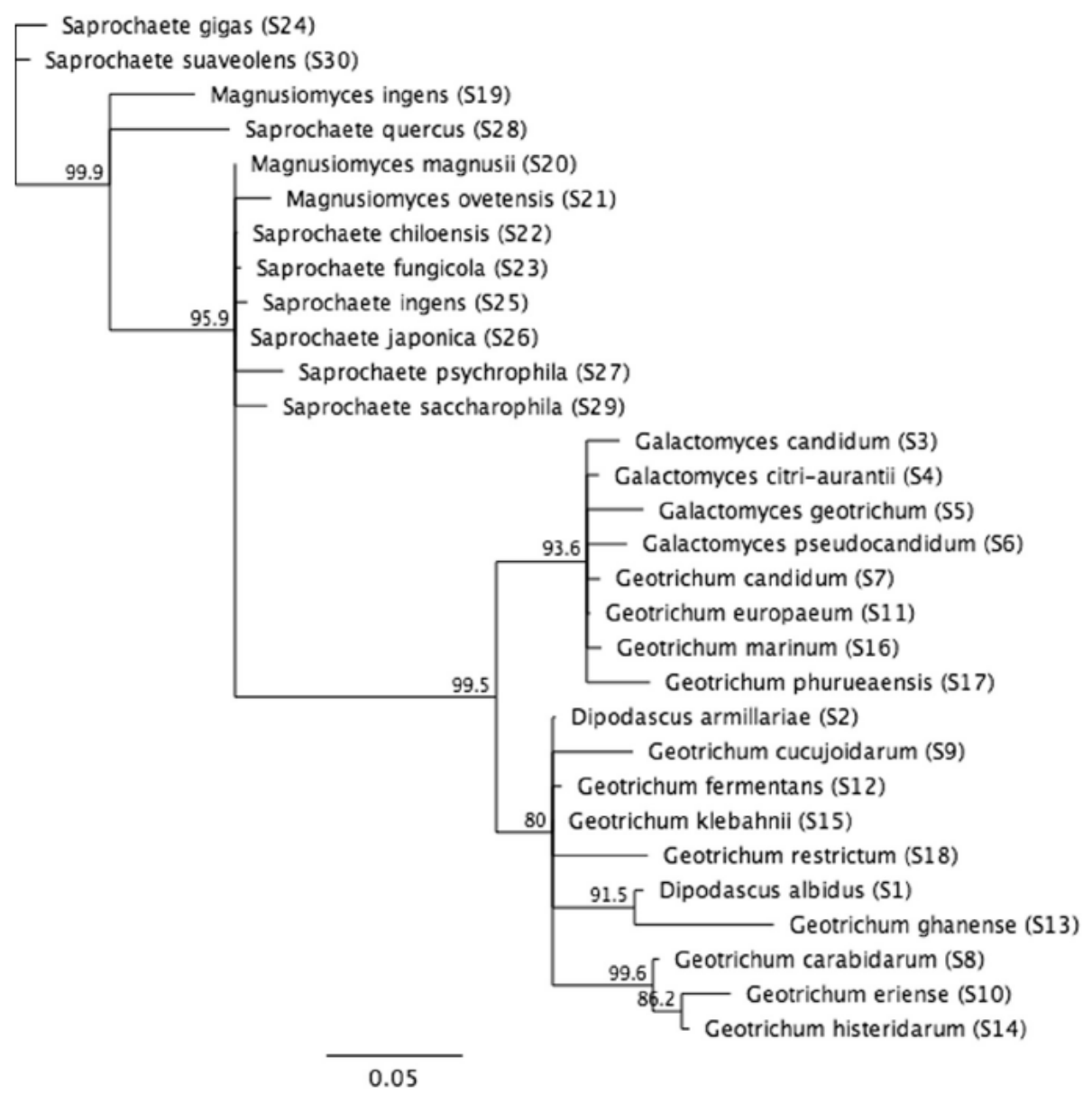

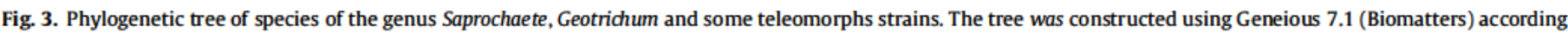

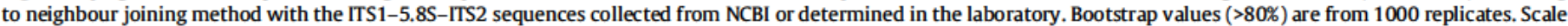
Bar represents the number of substitution per site.

ethyl 2 methylbut 2 enoate, ethyl 3 methylbut 2 enoate, 2 methylpropyl 2 methylbut 2 enoate, butyl 2 methylbut 2 enoate and 3 methylbutyl 2 methylbut 2 enoate. Some of them were also produced by other strains, namely Gal. geotrichum, Gal. pseudocan didus, G. europaeum, G. marinum, M. magnusii, S. gigas, S. quercus and $S$. suaveolens (Tables 1 and 2). Like S. suaveolens, G. marinum was found to produce 5 out of the 6 unsaturated esters. Ethyl 2 methylbut 2 enoate (ethyl tiglate) was produced by the 8 strains whereas some unsaturated esters like ethyl hex 2 enoate or butyl 2 methylbut 2 enoate (butyl tiglate) were produced by only one strain (G. marinum and S. suaveolens, respectively). Since four out of unsaturated esters were derived from 2 methylbutanoyl CoA or tiglyl CoA, we could argue according to Grondin, Shum Cheong Sing, Caro, de Billerbeck, et al. (2015), that these esters arose from the catabolism of isoleucine that partially halted at the dehydration step due to low enoyl CoA hydratase activity.

As reported in Table 2, G marinum can produce ethyl butanoate, ethyl but 2 enoate, ethyl hexanoate and ethyl hex 2 enoate, whereas S. suaveolens was limited to the production of the first two esters. This result may suggest that either S. suaveolens cannot further condense a acetyl CoA to elongate the fatty acid chain, or alternatively, butanoyl CoA is generated by the butanoate pathway that exist in human and some bacteria (http://www.genome.jp/ kegg/pathway.html) but to our knowledge had never been reported in fungi. This pathway can lead to either butanoate as well as to butanol. Together with intermediates in catabolism of isoleucine, this pathway may account for the production of butyl
2 methylbutanoate, butyl 2 methylbut 2 enoate and butyl 3 methylbutanoate.

\subsection{Comparison of the VOC profile of the strains by cluster and multivariate analysis and correlation with a taxonimical analysis}

Multivariate statistical analysis was used in order to group yeast species based on their flavouring characteristics. To this end, each strain was associated with variables described in Table 3. Cluster analysis (Fig. 1) and PCA analysis (Fig. 2) both suggested the occurrence of two main and two minor groups of yeasts. The first group seems to be characterized by strains which have a low ability to produce VOCs and the second by strains that pro duced mainly alcohols. Three strains, namely G. marinum, S. suave olens (group 3) and S. gigas (group 4) deviated from these groups because of their ability to produced esters (Fig. 2). Among group $2, G$. europaeum appeared to be the best producer of alcohols with $1164 \mu \mathrm{g} / \mathrm{L}$. In other experiments, we found Debaryomyces nepalen sis to be the best strain for alcohol production $(985 \mu \mathrm{g} / \mathrm{L}$ of alcohol was produced when tested in the same experimental conditions; Grondin, Shum Cheong Sing, Caro, Raherimandimby, et al., 2015). With a production of 15,30 and 32 different VOCs respectively, $S$. gigas, G. marinum and S. suaveolens were by far the best produc ers of esters, and more specifically unsaturated esters among all strains analyzed (Table 3). Geotrichum marinum has the particular ity to produce methyl esters like methyl 2 methylbutanoate and methyl 3 methylbutanoate (Table 2). 
The fact that 4 genera ( 8 species) produced these unsaturated compounds suggested phylogenetic belonging. Then, only Sapro chaete and related genera Geotrichum, Galactomyces and Magnu siomyces were found to produce these unsaturated esters, and in particular ethyl tiglate which could reach $113 \mathrm{mg} / \mathrm{l}$ in S. suaveolens culture (Grondin, Shum Cheong Sing, Caro, de Billerbeck, et al., 2015). The presence of unsaturated compounds in eight species of these neighbour genera indicated high metabolic similarities between these yeasts.

In order to establish a genomic link between these strains, a phylogenetic tree was generated using rDNA sequences either retrieved from the NCBI and GenBank databases or determined in our laboratory (Fig. 3). The internal transcribed spacer (ITS) region was selected because it is widely used to identify a broad range of different fungi (Schoch et al., 2012). As we can see, the strains under study seem very closed genetically ( 0.3 substitution per site between the most distant strains) and could explain the metabolic similarity for VOCS production (Fig. 3).

To summarize, this work was a first approach to study VOCS from the yeast of the genus Saprochaete, Geotrichum and close rel ative strains. Statistical analysis allowed us to classify the strains according to their flavour production and four groups of strains were highlighted by this approach. While we identified the Sapro chaete genus as exhibiting an unusual capacity to produce a large variety of unsaturated esters such as ethyl tiglate, we could not find any relationship between flavours profiles and genomic classi fication of these yeast strains, suggesting that the metabolic activ ities underlining the flavour production has been shaped by their ecological niche.

\section{Acknowledgments}

The authors thank gratefully the Regional Council of La Réunion (French overseas territory), the European Regional development Funds (ERDF) and the French Government for their financial and technical assistance through the QualiREG research network in Indian Ocean (www.qualireg.org). We also wish to thank the BIO FLAVOUR COST Action FA0907. The NCYC is a BBSRC supported National Capability.

\section{Appendix A. Supplementary data}

Supplementary data associated with this article can be found, in the online version, at http://dx.doi.org/10.1016/j.foodchem.2017. 06.009.

\section{References}

Ayed, L., Assas, N., Sayadi, S., \& Hamdi, M. (2005). Involvement of lignin peroxidase in the decolourization of black olive mill wastewaters by Geotrichum candidum. Letters in Applied Microbiology, 40(1), 7-11.

Berger, C., Khan, J. A., Molimard, P., Martin, N., \& Spinnler, H. E. (1999). Production of sulfur flavors by ten strains of Geotrichum candidum. Applied and Environmental Microbiology, 65(12), 5510-5514.

Bonnarme, P., Arfi, K., Dury, C., Helinck, S., Yvon, M., \& Spinnler, H. E. (2001). Sulfur compound production by Geotrichum candidum from L-methionine: Importance of the transamination step. FEMS Microbiology Letters, 205(2), 247-252.

Brabcova, J., Demianova, Z., Vondrasek, J., Jagr, M., Zarevucka, M., \& Palomo, J. M. (2013). Highly selective purification of three lipases from Geotrichum candidum 4013 and their characterization and biotechnological applications. Journal of Molecular Catalysis B: Enzymatic, 98, 62-72.

Buzzini, P., Martini, A., Cappelli, F., Pagnoni, U. M., \& Davoli, P. (2003). A study on volatile organic compounds (VOCs) produced by tropical ascomycetous yeasts. Antonie van Leeuwenhoek, 84(4), 301-311.

Camus, V., Thibault, M.-L., David, M., Gargala, G., Compagnon, P., Lamoureux, F. ... Leprêtre, S. (2014). Invasive Geotrichum clavatum fungal infection in an acute myeloid leukaemia patient: A case report and review. Mycopathologia, 1-6.

Cardinali, G., Antonielli, L., Corte, L., Roscini, L., Bagnetti, A., Pelliccia, C., \& Puddu, G. (2012). Kazachstania ichnusensis sp. nov., a diploid homothallic ascomycetous yeast from Sardinian lentisk rhizosphere. International Journal of Systematic and Evolutionary Microbiology, 62(Pt 3), 722-727.
Damasceno, S., Cereda, M. P., Pastore, G. M., \& Oliveira, J. G. (2003). Production of volatile compounds by Geotrichum fragrans using cassava wastewater as substrate. Process Biochemistry, 39(4), 411-414.

De Hoog, S. G., \& Smith, M. T. (2004). Ribosomal gene phylogeny and species delimitation in Geotrichum and its teleomorphs. Studies in Mycology, 50, 489-515.

De Hoog, S. G., \& Smith, M. T. (2011a). Chapter 91 - Geotrichum link. In C. P. Kurtzman, J. W. Fell, \& T. Boekhout (Eds.), The Yeasts (5th ed., pp. 1279-1286). London: Elsevier.

De Hoog, S. G., \& Smith, M. T. (2011b). Chapter 97 - Saprochaete Coker \& Shanor ex D.T.S. Wagner \& Dawes (1970). In C. P. Kurtzman, J. W. Fell, \& T. Boekhout (Eds.) The yeasts (5th ed., pp. 1317-1327). London: Elsevier.

De Oliveira, S. M. M., Gomes, S. D., Sene, L., Machado, S. R., Coelho, A. C. B., Cereda, M. P., ... Piechontcoski, J. (2013). Production of 2-phenylethanol by Geotrichum fragrans, Saccharomyces cerevisiae and Kluyveromyces marxianus in cassava wastewater. Journal of Food, Agriculture E' Environment, 11(2), 158-163.

Farbood, M. I., Morris, J. A., \& Seitz, E. W. (1987). Preparation of naturally-occurring C2-C5 alkyl esters of C4-C5 carboxylic acids by means of fermentation of C5-C6 amino acids in the presence of C2-C5 alcohols. US patent US4657862 A.

Fischer, K.-H., Senser, F., \& Grosch, W. (1983). Aromabildung durch Mikroorganismen. Zeitschrift Für Lebensmittel-Untersuchung Und Forschung, 177 (5), 336-338.

Frisvad, J. C., Andersen, B., \& Thrane, U. (2008). The use of secondary metabolite profiling in chemotaxonomy of filamentous fungi. Mycological Research, 112(2), 231-240.

Garcia-Ruiz, J. C., Lopez-Soria, L., Olazabal, I. Amutio, E., Arrieta-Aguirre, I., VelascoBenito, V., ... Moragues, M. D. (2013). Invasive infections caused by Saprochaete capitata in patients with haematological malignancies: Report of five cases and review of the antifungal therapy. Revista Iberoamericana de Micología, 30, $248-255$.

Grondin, E., Shum Cheong Sing, A., Caro, Y., de Billerbeck, G. M., François, J. M., \& Petit, T. (2015a). Physiological and biochemical characteristics of ethyl tiglate production pathway in the yeast Saprochaete suaveolens. Yeast, 32(1), 57-66.

Grondin, E., Shum Cheong Sing, A., Caro, Y., Raherimandimby, M., Randrianierenana, A. L., James, S., ... Petit, T. (2015b). A comparative study on the potential of epiphytic yeasts isolated from tropical fruits to produce flavoring compounds. International Journal of Food Microbiology, 203, 101-108.

Hang, Y. D., \& Woodams, E. E. (1990). Lipase production by Geotrichum candidum from sauerkraut brine. World Journal of Microbiology E' Biotechnology, 6(4), $418-421$.

Hazelwood, L. A., Daran, J.-M., van Maris, A. J. A., Pronk, J. T., \& Dickinson, J. R. (2008). The Ehrlich pathway for fusel alcohol production: A century of research on Saccharomyces cerevisiae metabolism. Applied and Environmental Microbiology, 74(8), 2259-2266.

James, S. A., Barriga, E. J. C., Barahona, P. P., Harrington, T. C., Lee, C.-F., Bond, C. J., \& Roberts, I. N. (2014). Wickerhamomyces arborarius f.a., sp. nov., an ascomycetous yeast species found in arboreal habitats on three different continents. International Journal of Systematic and Evolutionary Microbiology, 64(Pt 3), 1057-1061.

Jollivet, N., Chataud, J., Vayssier, Y., Bensoussan, M., \& Belin, J.-M. (1994). Production of volatile compounds in model milk and cheese media by eight strains of Geotrichum candidum Link. Journal of Dairy Research, 61(02), 241-248.

Larsen, T. O., \& Frisvad, J. C. (1995). Chemosystematics of Penicillium based on profiles of volatile metabolites. Mycological Research, 99(10), 1167-1174.

Liu, S.-Q., Holland, R., \& Crow, V. L. (2004). Esters and their biosynthesis in fermented dairy products: A review. International Dairy Journal, 14(11), 923-945.

Liu, Z.-Y., Yao, Y.-J., Liang, Z. Q., Liu, A.-Y., Pegler, D. N., \& Chase, M. W. (2001) Molecular evidence for the anamorph-teleomorph connection in Cordyceps sinensis. Mycological Research, 105(07), 827-832.

Maggio-Hall, L. A., \& Keller, N. P. (2004). Mitochondrial $\beta$-oxidation in Aspergillus nidulans. Molecular Microbiology, 54(5), 1173-1185.

Marcellino, N., Beuvier, E., Grappin, R., Guéguen, M., \& Benson, D. R. (2001). Diversity of Geotrichum candidum strains isolated from traditional cheesemaking fabrications in France. Applied and Environmental Microbiology, 67(10), 4752-4759.

Neto, R. S., Pastore, G. M., \& Macedo, G. A. (2004). Biocatalysis and biotransformation producing $\gamma$-decalactone. Journal of Food Science, 69(9), 677-680.

Nielsen, D. S., Jakobsen, M., \& Jespersen, L. (2010). Candida halmiae sp. nov., Geotrichum ghanense sp. nov. and Candida awuaii sp. nov., isolated from Ghanaian cocoa fermentations. International Journal of Systematic and Evolutionary Microbiology, 60(6), 1460-1465.

Saerens, S. M. G., Delvaux, F. R., Verstrepen, K. J., \& Thevelein, J. M. (2010). Production and biological function of volatile esters in Saccharomyces cerevisiae. Microbial Biotechnology, 3(2), 165-177.

Schoch, C. L., Seifert, K. A., Huhndorf, S., Robert, V., Spouge, J. L., Levesque, C. A., ... Schindel, D. (2012). Nuclear ribosomal internal transcribed spacer (ITS) region as a universal DNA barcode marker for Fungi. Proceedings of the National Academy of Sciences, 109(16), 6241-6246.

Schrader, J. (2007). Microbial flavour production. In R. G. Berger (Ed.), Flavours and fragrances (pp. 507-574). Berlin Heidelberg: Springer.

Shimizu, S., Kataoka, M., Kizaki, N., \& Yasohara, Y. (2004). Incubating ashbya with 2chloro-1-(3'-chlorophenyl)ethanone, allowing sterospecific reduction, recovering ( $R$ )-2-chloro-1-(3'-chlorophenyl)ethanol; raw materials for medicine. US patent US20040219658 A1. 
Sinha, N. (2007). Handbook of food products manufacturing, 2 volume set. John Wiley \& Sons.

Suh, S.-O., \& Blackwell, M. (2006). Three new asexual arthroconidial yeasts, Geotrichum carabidarum sp. nov., Geotrichum histeridarum sp. nov., and Geotrichum cucujoidarum sp. nov., isolated from the gut of insects. Mycological Research, 110(Pt. 2), 220-228.

Ugliano, M., \& Henschke, P. A. (2009). Yeasts and wine flavour. In M. V. MorenoArribas \& M. C. Polo (Eds.), Wine chemistry and biochemistry (pp. 313-392). New York: Springer.
Urubschurov, V., Janczyk, P., Souffrant, W.-B., Freyer, G., \& Zeyner, A. (2011) Establishment of intestinal microbiota with focus on yeasts of unweaned and weaned piglets kept under different farm conditions. FEMS Microbiology Ecology, 77(3), 493-502.

Wu, Q., Xu, Y., \& Chen, L. (2012). Diversity of yeast species during fermentative process contributing to Chinese Maotai-flavour liquor making. Letters in Applied Microbiology, 55(4), 301-307. 